\title{
Pacific
}

Journal of

Mathematics

\section{ON BACH FLAT WARPED PRODUCT EINSTEIN MANIFOLDS}

Qiang Chen and ChenXu He 


\title{
ON BACH FLAT WARPED PRODUCT EINSTEIN MANIFOLDS
}

\author{
QIANG Chen AND ChenXu He
}

\begin{abstract}
We show that a compact warped product Einstein manifold with vanishing Bach tensor of dimension $n \geq 4$ is either Einstein or a finite quotient of a warped product with an $(n-1)$-dimensional Einstein fiber. The fiber has constant curvature if $n=4$.
\end{abstract}

\section{Introduction}

Let $\lambda, m \in \mathbb{R}$ be constants. A $(\lambda, n+m)$-Einstein manifold is a complete Riemannian manifold with a smooth function $f$ that satisfies the $(\lambda, n+m)$-Einstein equation

$$
\operatorname{Ric}_{f}^{m}=\operatorname{Ric}+\text { Hess } f-\frac{1}{m} d f \otimes d f=\lambda g .
$$

When $m$ is a positive integer, $(\lambda, n+m)$-Einstein metrics are exactly those $n$ dimensional manifolds which are the base of an $n+m$ dimensional Einstein warped product, i.e., $\left(M \times F^{m}, g+e^{-2 f / m} g_{F}\right)$ is an Einstein manifold with Einstein constant $\lambda$, where $\left(F^{m}, g_{F}\right)$ is another Einstein manifold [Besse 1987].

We call Ric ${ }_{f}^{m}$ in (1-1) the m-Bakry-Emery tensor. Lower bounds on this tensor are given by various comparison theorems for the measure $e^{-f} d \mathrm{vol}_{g}$; see, for example, [Villani 2009, Part II; Wei and Wylie 2009]. In view of these comparison theorems, the $(\lambda, n+m)$-Einstein equation is the natural Einstein condition of having a constant $m$-Bakry-Emery Ricci tensor. The study of $(\lambda, n+1)$-Einstein metrics often needs special techniques, so we do not consider these metrics here: we assume $m \neq 1$ throughout this paper. Taking $m \rightarrow \infty$, one also obtains the gradient Ricci soliton equation

$$
\text { Ric }+ \text { Hess } f=\lambda g \text {. }
$$

We could then also call a gradient Ricci soliton a $(\lambda, \infty)$-Einstein manifold. Ricci solitons have been studied because of their connection to Ricci flow and because they are a natural generalization of Einstein manifolds. We refer to the survey paper [Cao 2010] for recent progress on this subject.

MSC2000: 53B20, 53C21, 53C25.

Keywords: warped product metric, Einstein manifold, Bach flat, Bakry-Emery tensor. 
In a series of papers with P. Petersen and W. Wylie, the second author studied warped product Einstein manifolds under various curvature and symmetry conditions [He et al. 2010; 2011; 2012]. Many interesting results on gradient Ricci solitons have also been obtained on warped product Einstein manifolds. In [He et al. 2010] we found nontrivial examples of manifolds on homogeneous spaces that are neither Einstein nor products of Einstein manifolds. This contrasts with the gradient Ricci soliton case, where all homogeneous gradient Ricci solitons are Einstein or products of Einstein manifolds.

In this paper, we consider an interesting class of complete warped product Einstein manifolds: those with a vanishing Bach tensor. This well-known tensor was first introduced by R. Bach [1921] to study conformal relativity. On any Riemannian manifold $\left(M^{n}, g\right)(n \geq 4)$, the Bach tensor is defined by

$B(X, Y)=\frac{1}{n-3}\left(\nabla_{E_{i}, E_{j}}^{2} W\right)\left(X, E_{i}, E_{j}, Y\right)+\frac{1}{n-2} \operatorname{Ric}\left(E_{i}, E_{j}\right) W\left(X, E_{i}, E_{j}, Y\right)$.

Here, $\left\{E_{i}\right\}_{i=1}^{n}$ is an orthonormal frame, $\nabla_{E_{i}, E_{j}}^{2}$ is the covariant derivative of tensors, and $W$ is the Weyl curvature tensor. If the manifold is Einstein or locally conformally flat, the Bach tensor vanishes. The dimension 4 is most interesting since on any compact 4-manifold $\left(M^{4}, g\right)$, Bach flat metrics are precisely the critical points of the conformally invariant functional on the space of metrics

$$
\mathscr{W}(g)=\int_{M}\left|W_{g}\right|^{2} d \operatorname{vol}_{g},
$$

where $W_{g}$ is the Weyl tensor of the metric $g$. Other than Einstein and locally conformally flat metrics, there are two more classes of compact 4-manifolds with a vanishing Bach tensor: metrics that are locally conformal to an Einstein one, and half conformally flat metrics (self-dual or anti-self-dual) if $M^{4}$ is orientable. The aim of this paper is to show that a stronger converse of warped product Einstein metrics holds. The proof is motivated by a recent corresponding result on gradient Ricci solitons [Cao and Chen 2011].

Theorem 1.1. Suppose $\left(M^{4}, g, f\right)$ is a compact $(\lambda, 4+m)$-Einstein manifold with $m \neq 0,1$, or -2 . If the Bach tensor $B$ vanishes everywhere, then $M$ is locally conformally flat.

Theorem 1.1 is a direct consequence of the following more general result combined with some early results in [He et al. 2012].

Theorem 1.2. Suppose $\left(M^{n}, g, f\right)(n \geq 4)$ is a compact $(\lambda, n+m)$-Einstein manifold with $m \neq 0,1$, or $2-n$. If the Bach tensor $B$ vanishes everywhere, then $M$ has a harmonic Weyl tensor and $W(X, Y, Z, \nabla f)=0$ for any vector fields $X, Y$, and $Z$.

Remark 1.3. Theorem 1.2 is analogous to [Cao and Chen 2011, Theorem 5.1]. 
Remark 1.4. In the case where $m=2-n$, our argument breaks down, since some coefficient vanishes in the key identity (3-1). On the other hand, it is observed in [Catino et al. 2011] that in this case, a $(\lambda, n+(2-n))$-Einstein metric is globally conformally Einstein. In particular, it has a vanishing Bach tensor when $n=4$.

Remark 1.5. Böhm [1998] constructed compact, rotationally symmetric $(\lambda, n+m)$ Einstein metrics on $\mathbb{S}^{n}$ for $n=3,4,5,6,7$ that are not Einstein. This is in sharp contrast to the gradient Ricci solitons. These examples also show that our conclusion in dimension 4 cannot be strengthened.

Remark 1.6. Theorem 1.1 was first obtained by G. Catino [2012] under a stronger assumption that $\left(M^{4}, g\right)$ is half conformally flat.

If $m$ is positive, then from [Kim and Kim 2003] and the comparison theorem of $m$-Bakry-Emery tensors in [Qian 1997, Theorem 5], a $(\lambda, n+m)$-Einstein manifold is compact if and only if $\lambda>0$. Using [He et al. 2012, Theorem 1.5], the global classification of warped product Einstein manifolds with harmonic Weyl tensor, and $W(\nabla f, \cdot, \cdot, \nabla f)=0$, Theorem 1.2 has the following corollary:

Corollary 1.7. Let $m \neq 1$ be a positive number. Suppose that $\left(M^{n}, g, f\right)(n \geq 4)$ is a simply connected $(\lambda, n+m)$-Einstein manifold with $\lambda>0$ and has a vanishing Bach tensor. Then $\left(M^{n}, g, f\right)$ is either

(1) Einstein with constant function $f$, or

(2) $g=d t^{2}+\psi^{2}(t) g_{L}$ and $f=f(t)$, where $g_{L}$ is Einstein with nonnegative Ricci curvature, and has constant curvature if $n=4$.

Remark 1.8. In the proof of [He et al. 2012, Theorem 1.5], the authors made the assumption that $m>1$. In fact, the whole argument carries over for the case when $0<m<1$.

Remark 1.9. Since $m$ can be an arbitrary constant in our definition of a $(\lambda, n+m)$ Einstein manifold, we would like to discuss the case when $m<0$. In this case, a $(\lambda, n+m)$-Einstein manifold with positive $\lambda$ is not necessarily compact, as the proof of the comparison theorem [Qian 1997, Theorem 5] is no longer valid. On the other hand, if we assume further that $m \neq 2-n$, the argument of the local classification result in [He et al. 2012, Theorem 7.9] carries over (see also [Catino et al. 2011] and Remark 3.4). The global result in [He et al. 2012, Theorem 1.5] also holds, except for the statement that $\left(L, g_{L}\right)$ has nonnegative Ricci curvature when $\lambda \geq 0$. The argument of that statement relies on the positivity of $m$ (see [He et al. 2012, Theorem 7.10]).

The paper is organized as follows. In Section 2, we recall definitions and basic properties of Bach, Cotton, and Weyl tensors, and the $D$-tensor defined in [Cao and Chen 2011]. We also list some relevant properties of warped product Einstein 
metrics. In Section 3, we show how the $D$-tensor characterizes the geometry of the level set of $f-$ see Proposition 3.3. In Section 4, we prove Theorem 1.1 and Theorem 1.2.

\section{Preliminaries}

In this section, we set up our notation and recall some well-known facts on warped product Einstein manifolds. For more detail, see for example [He et al. 2012] and references therein.

We use the convention that the Riemann curvature tensor $R(X, Y, Y, X)$ has the same sign as the sectional curvature of the 2-plane spanned by $X$ and $Y$. For $n \geq 4$, the Weyl curvature tensor is defined as

$$
R=W+\frac{2}{n-2} \operatorname{Ric} \odot g-\frac{\text { scal }}{(n-1)(n-2)} g \odot g,
$$

where, for two symmetric $(0,2)$-tensors $s$ and $r$, we define the Kulkarni-Nomizu product $s \odot r$ to be the $(0,4)$-tensor

$$
\begin{aligned}
& (s \odot r)(X, Y, Z, W) \\
& \quad=\frac{1}{2}(r(X, W) s(Y, Z)+r(Y, Z) s(X, W)-r(X, Z) s(Y, W)-r(Y, W) s(X, Z)) .
\end{aligned}
$$

Recall that for any $X, Y \in T M$, the Bach tensor $B$ is the symmetric $(0,2)$-tensor defined by

$$
\begin{aligned}
B(X, Y)=\frac{1}{n-3} \sum_{i, j}\left(\nabla_{E_{i}, E_{j}}^{2} W\right) & \left(X, E_{i}, E_{j}, Y\right) \\
& +\frac{1}{n-2} \sum_{i, j} \operatorname{Ric}\left(E_{i}, E_{j}\right) W\left(X, E_{i}, E_{j}, Y\right),
\end{aligned}
$$

where $\left\{E_{i}\right\}_{i=1}^{n}$ is an orthonormal frame and $\nabla_{E_{i}, E_{j}}^{2} W$ is the covariant derivative of the Weyl tensor.

The Schouten tensor is the $(0,2)$-tensor

$$
S=\operatorname{Ric}-\frac{\text { scal }}{2(n-1)} g
$$

and the Cotton tensor $C$ is defined as

$$
C(X, Y, Z)=\left(\nabla_{X} S\right)(Y, Z)-\left(\nabla_{Y} S\right)(X, Z) \text { for any } X, Y, Z \in T M \text {. }
$$

Using the fact that $(\operatorname{div} R)(X, Y, Z)=\left(\nabla_{X} \operatorname{Ric}\right)(Y, Z)-\left(\nabla_{Y} \operatorname{Ric}\right)(X, Z)$, we have (2-2) $\quad C(X, Y, Z)$

$$
=(\operatorname{div} R)(X, Y, Z)-\frac{1}{2(n-1)}\left(\left(\nabla_{X} \operatorname{scal}\right) g(Y, Z)-\left(\nabla_{Y} \operatorname{scal}\right) g(X, Z)\right) .
$$


Definition 2.1. A Riemannian manifold $\left(M^{n}, g\right)$ has a harmonic Weyl tensor if the Cotton tensor vanishes.

Remark 2.2. For $n \geq 4$, the Cotton tensor is, up to a constant factor, the divergence of the Weyl tensor

$$
C(X, Y, Z)=\frac{n-2}{n-3}(\operatorname{div} W)(X, Y, Z) \quad \text { for any } X, Y, Z \in T M .
$$

So we can rewrite the Bach tensor as

$$
B(X, Y)=\frac{1}{n-2}\left(\sum_{i}\left(\nabla_{E_{i}} C\right)\left(E_{i}, X, Y\right)+\sum_{i, j} \operatorname{Ric}\left(E_{i}, E_{j}\right) W\left(X, E_{i}, E_{j}, Y\right)\right),
$$

where $\left\{E_{i}\right\}_{i=1}^{n}$ is an orthonormal frame.

Remark 2.3. If $n=3$, then $W=0$, and thus a harmonic Weyl tensor is equivalent to $\left(M^{3}, g\right)$ being locally conformally flat. If $n \geq 4$, then $M$ has a harmonic Weyl tensor if and only if $\operatorname{div} W=0$, and $M$ is locally conformally flat if and only if $W=0$.

On a $(\lambda, n+m)$-Einstein manifold $(M, g, f)$ for any $X, Y, Z \in T M$ we define the $D$-tensor, which is identical to the one in [Cao and Chen 2011] for Ricci solitons, as follows:

$$
\begin{aligned}
D(X, Y, Z)= & \frac{1}{(n-1)(n-2)}(\operatorname{Ric}(X, \nabla f) g(Y, Z)-\operatorname{Ric}(Y, \nabla f) g(X, Z)) \\
& +\frac{1}{n-2}(\operatorname{Ric}(Y, Z) g(X, \nabla f)-\operatorname{Ric}(X, Z) g(Y, \nabla f)) \\
& -\frac{\operatorname{scal}}{(n-1)(n-2)}(g(X, \nabla f) g(Y, Z)-g(Y, \nabla f) g(X, Z)) .
\end{aligned}
$$

Both $C$ and $D$ are skew-symmetric in their first two indices and trace-free in any two indices:

$$
\begin{aligned}
& C(X, Y, Z)=-C(Y, X, Z), \quad \sum_{i} C\left(E_{i}, E_{i}, X\right)=\sum_{i} C\left(E_{i}, X, E_{i}\right)=0, \\
& D(X, Y, Z)=-D(Y, X, Z), \quad \sum_{i} D\left(E_{i}, E_{i}, X\right)=\sum_{i} D\left(E_{i}, X, E_{i}\right)=0 .
\end{aligned}
$$

Next we recall some properties of warped product Einstein manifolds; the proofs can be found in [He et al. 2012]. The function $\rho$ is defined by

$$
\text { scal }=(n-1) \lambda-(m-1) \rho .
$$

Note that a $(\lambda, n+1)$-Einstein manifold has constant scalar curvature $(n-1) \lambda$. The modified Ricci and Riemann curvature tensors are defined by

$$
P=\operatorname{Ric}+\rho g
$$


and

$$
Q=R+\frac{2}{m} \operatorname{Ric} \odot g-\frac{\lambda+\rho}{m} g \odot g=R+\frac{2}{m} P \odot g+\frac{\rho-\lambda}{m} g \odot g .
$$

Proposition 2.4. Let $(M, g, f)$ be a $(\lambda, n+m)$-Einstein manifold with $m \neq 1$. Then

$$
\begin{aligned}
& P(\nabla f)=-\frac{m}{2} \nabla \rho, \text { or equivalently } \operatorname{Ric}(\nabla f)=-\frac{m}{2} \nabla \rho+\rho \nabla f ; \\
& (\operatorname{div} R)=Q(X, Y, Z, \nabla f)-\frac{1}{m}(g \odot g)(X, Y, Z, P(\nabla f)) .
\end{aligned}
$$

Equation (2-6) is (3.12) in [Case et al. 2011], and (2-7) was shown in [He et al. 2012, Proposition 6.3].

\section{The covariant 3-tensor $D$}

In this section, we extend some known results regarding the 3-tensor $D$ from gradient Ricci solitons to warped product Einstein manifolds. Since the $(\lambda, n+m)$-Einstein equation (1-1) contains the extra term in $d f \otimes d f$, we provide the calculations in detail, though we essentially follow proofs in [Cao and Chen 2011; 2012].

For gradient Ricci solitons, the $D$ tensor relates the Cotton tensor and Weyl tensor in the following way (see [Cao and Chen 2011, Lemma 3.1]):

$$
C(X, Y, Z)=D(X, Y, Z)+W(X, Y, Z, \nabla f) \text { for any } X, Y, Z \in T M .
$$

For warped product manifolds, we have a similar relation for these three tensors. (The case $n=4$ was given in [Catino 2012].)

Lemma 3.1. Suppose $\left(M^{n}, g, f\right)$ is a $(\lambda, n+m)$-Einstein manifold. The Cotton tenor $C, D$-tensor, and Weyl tensor $W$ satisfy the identity

(3-1) $C(X, Y, Z)=W(X, Y, Z, \nabla f)+\frac{m+n-2}{m} D(X, Y, Z) \quad$ for $X, Y, Z \in T M$.

Proof. From the formula (2-7) for $\operatorname{div} R$, the definition of $Q$-tensor, and the decomposition curvature tensor $R$, we have (with a dash standing for the three first arguments $X, Y, Z$ )

$(\operatorname{div} R)(-)$

$$
\begin{aligned}
& =Q(-, \nabla f)-\frac{1}{m}(g \odot g)(-, P(\nabla f)) \\
& =R(-, \nabla f)+\frac{2}{m}(\operatorname{Ric} \odot g)(-, \nabla f)-\frac{\lambda+\rho}{m}(g \odot g)(-, \nabla f)-\frac{1}{m}(g \odot g)(-, P(\nabla f)) \\
& =W(-, \nabla f)+\frac{2}{n-2}(\operatorname{Ric} \odot g)(-, \nabla f)-\frac{(n-1) \lambda-(m-1) \rho}{(n-1)(n-2)}(g \odot g)(-, \nabla f) \\
& \quad+\frac{2}{m}(\operatorname{Ric} \odot g)(-, \nabla f)-\frac{\lambda+\rho}{m}(g \odot g)(-, \nabla f)-\frac{1}{m}(g \odot g)(-, P(\nabla f))
\end{aligned}
$$




$$
\begin{aligned}
=W(-, \nabla f)-\frac{1}{m}(g \odot g)(-, P(\nabla f))+\frac{2(m+n-2)}{m(n-2)}(\operatorname{Ric} \odot g)(-, \nabla f) \\
-\frac{(n-1)(m+n-2) \lambda+((n-1)(n-2)-m(m-1)) \rho}{m(n-1)(n-2)}(g \odot g)(-, \nabla f) .
\end{aligned}
$$

Using the fact that $P=$ Ric $-\rho g$, we have

$(\operatorname{div} R)(X, Y, Z)$

$$
\begin{aligned}
= & W(X, Y, Z, \nabla f)+\frac{1}{n-2}(\operatorname{Ric}(X, \nabla f) g(Y, Z)-\operatorname{Ric}(Y, \nabla f) g(X, Z)) \\
& +\frac{m+n-2}{m(n-2)}(\operatorname{Ric}(Y, Z) g(X, \nabla f)-\operatorname{Ric}(X, Z) g(Y, \nabla f)) \\
& -\frac{(n-1)(m+n-2) \lambda-m(m-1) \rho}{m(n-1)(n-2)}(g(X, \nabla f) g(Y, Z)-g(Y, \nabla f) g(X, Z)) .
\end{aligned}
$$

From the formula (2-6) for $\operatorname{Ric}(\nabla f)$, we have

$(\operatorname{div} R)(X, Y, Z, \nabla f)-W(X, Y, Z, \nabla f)$

$$
\begin{aligned}
= & -\frac{m}{2(n-2)}\left(\left(\nabla_{X} \rho\right) g(Y, Z)-\left(\nabla_{Y} \rho\right) g(X, Z)\right) \\
& +\frac{m+n-2}{m(n-2)}(\operatorname{Ric}(Y, Z) g(X, \nabla f)-\operatorname{Ric}(X, Z) g(Y, \nabla f)) \\
& -\frac{m+n-2}{m(n-1)(n-2)}((n-1) \lambda-m \rho)(g(X, \nabla f) g(Y, Z)-g(Y, \nabla f) g(X, Z)) .
\end{aligned}
$$

From the defining equation (2-2) of the Cotton tensor $C$ and using scal $=(n-1) \lambda-$ $(m-1) \rho$, we have

$$
C(X, Y, Z)=(\operatorname{div} R)(X, Y, Z)+\frac{m-1}{2(n-1)}\left(\left(\nabla_{X} \rho\right) g(Y, Z)-\left(\nabla_{Y} \rho\right) g(X, Z)\right),
$$

and then

$$
\begin{aligned}
\frac{m}{m+n-2}(C(X, Y, Z) & -W(X, Y, Z, \nabla f)) \\
= & -\frac{m}{2(n-1)(n-2)}\left(\left(\nabla_{X} \rho\right) g(Y, Z)-\left(\nabla_{Y} \rho\right) g(X, Z)\right) \\
& +\frac{1}{n-2}(\operatorname{Ric}(Y, Z) g(X, \nabla f)-\operatorname{Ric}(X, Z) g(Y, \nabla f)) \\
& -\frac{(n-1) \lambda-m \rho}{(n-1)(n-2)}(g(X, \nabla f) g(Y, Z)-g(Y, \nabla f) g(X, Z)),
\end{aligned}
$$

which is exactly equal to $D(X, Y, Z)$ by the formula of $\operatorname{Ric}(\nabla f)$.

For gradient Ricci solitons, one amazing fact is that the norm of the $D$-tensor is linked to the geometry of the level set of the potential function $f$ (see [Cao 
and Chen 2012, (4.5); Cao and Chen 2011, Lemma 3.2]). We have the following extension to warped product Einstein manifolds.

Lemma 3.2. Suppose $\left(M^{n}, g, f\right)$ is a $(\lambda, n+m)$-Einstein manifold. Let $\Sigma^{n-1}$ be a level set of $f$ with $\nabla f(p) \neq 0$, and let $h_{a b}(a, b=2, \ldots, n)$ and $H=(n-1) \sigma$ be its second fundamental form and mean curvature, respectively. Then we have

$$
|D|^{2}=\frac{2|\nabla f|^{4}}{(n-2)^{2}} \sum_{a, b=2}^{n}\left|h_{a b}-\sigma g_{a b}\right|^{2}+\frac{m^{2}}{2(n-1)(n-2)(m-1)^{2}}\left|\nabla^{\Sigma} \mathrm{scal}\right|^{2}
$$

where $\left|\nabla^{\Sigma} \mathrm{scal}\right|^{2}=|\nabla \mathrm{scal}|^{2}-(\nabla \mathrm{scal} \cdot(\nabla f /|\nabla f|))^{2}$.

Proof. Let $\left\{e_{i}\right\}_{i=1}^{n}$ be an orthonormal frame with $e_{1}=\nabla f /|\nabla f|$ at the point where $\nabla f$ is nonzero. The second fundamental form $h_{a b}$ and the mean curvature $H$ of the level hypersurface $\Sigma$ are given by

$$
\begin{aligned}
h_{a b} & =g\left(\nabla_{e_{a}} \frac{\nabla f}{|\nabla f|}, e_{b}\right)=\frac{1}{|\nabla f|} \nabla_{e_{a}} \nabla_{e_{b}} f=\frac{1}{|\nabla f|}\left(\lambda g_{a b}-\operatorname{Ric}\left(e_{a}, e_{b}\right)\right), \\
H & =\frac{1}{|\nabla f|}\left((n-1) \lambda-\operatorname{scal}+\operatorname{Ric}\left(e_{1}, e_{1}\right)\right) .
\end{aligned}
$$

So we have

$$
\begin{aligned}
& \sum_{a, b=2}^{n}\left|h_{a b}\right|^{2}=\frac{1}{|\nabla f|^{2}} \sum_{a, b=2}^{n}\left|\lambda g_{a b}-\operatorname{Ric}\left(e_{a}, e_{b}\right)\right|^{2} \\
&=\frac{1}{|\nabla f|^{2}}\left((n-1) \lambda^{2}-2 \lambda\left(\operatorname{scal}-\operatorname{Ric}\left(e_{1}, e_{1}\right)\right)+\sum_{a, b=2}^{n}\left|\operatorname{Ric}\left(e_{a}, e_{b}\right)\right|^{2}\right), \\
& H^{2}=\frac{1}{|\nabla f|^{2}}\left((n-1)^{2} \lambda^{2}-2(n-1) \lambda\left(\operatorname{scal}-\operatorname{Ric}\left(e_{1}, e_{1}\right)+\left(\operatorname{scal}-\operatorname{Ric}\left(e_{1}, e_{1}\right)\right)^{2}\right)\right) .
\end{aligned}
$$

From $\operatorname{Ric}(\nabla f)=\rho \nabla f-\frac{m}{2} \nabla \rho$, it follows that

$$
\begin{aligned}
& R_{11}=\operatorname{Ric}\left(e_{1}, e_{1}\right)=\rho-\frac{m}{2|\nabla f|^{2}} \nabla \rho \cdot \nabla f, \\
& R_{1 a}=\operatorname{Ric}\left(e_{1}, e_{a}\right)=-\frac{m}{2|\nabla f|} \nabla_{a} \rho .
\end{aligned}
$$

So we have

$$
\begin{aligned}
& \sum_{a, b=2}^{n}\left|h_{a b}-\sigma g_{a b}\right|^{2} \\
& \quad=\frac{1}{|\nabla f|^{2}}|\mathrm{Ric}|^{2}-\frac{2}{|\nabla f|^{2}} \sum_{a=2}^{n} R_{1 a}^{2}-\frac{1}{|\nabla f|^{2}} R_{11}^{2}-\frac{1}{(n-1)|\nabla f|^{2}}\left(\mathrm{scal}-R_{11}\right)^{2},
\end{aligned}
$$


where

$$
\begin{aligned}
-\frac{2}{|\nabla f|^{2}} \sum_{a=2}^{n} R_{1 a}^{2} & =-\frac{m^{2}}{2|\nabla f|^{4}}|\nabla \rho|^{2}+\frac{m^{2}}{2|\nabla f|^{6}}(\nabla \rho \cdot \nabla f)^{2}, \\
-\frac{1}{|\nabla f|^{2}} R_{11}^{2} & =-\frac{1}{|\nabla f|^{2}} \rho^{2}+\frac{m \rho}{|\nabla f|^{4}} \nabla \rho \cdot \nabla f-\frac{m^{2}}{4|\nabla f|^{6}}(\nabla \rho \cdot \nabla f)^{2}, \\
\text { scal }-R_{11} & =(n-1) \lambda-m \rho+\frac{m}{2|\nabla f|^{2}} \nabla \rho \cdot \nabla f,
\end{aligned}
$$

and

$$
\begin{aligned}
-\frac{1}{(n-1)|\nabla f|^{2}}\left(\operatorname{scal}-R_{11}\right)^{2} & =-\frac{(n-1) \lambda^{2}}{|\nabla f|^{2}}-\frac{m^{2} \rho^{2}}{(n-1)|\nabla f|^{2}}+\frac{2 m \lambda \rho}{|\nabla f|^{2}} \\
& -\frac{m^{2}}{4(n-1)|\nabla f|^{6}}(\nabla \rho \cdot \nabla f)^{2}+\frac{m(m \rho-(n-1) \lambda)}{(n-1)|\nabla f|^{4}} \nabla \rho \cdot \nabla f .
\end{aligned}
$$

Adding them together yields

$$
\begin{aligned}
& \sum_{a, b=2}^{n}\left|h_{a b}-\sigma g_{a b}\right|^{2} \\
& =\frac{1}{|\nabla f|^{2}}|\mathrm{Ric}|^{2}-\frac{m^{2}}{2|\nabla f|^{4}}|\nabla \rho|^{2}+\frac{m^{2}(n-2)}{4(n-1)|\nabla f|^{6}}(\nabla \rho \cdot \nabla f)^{2} \\
& \quad+\frac{m((m+n-1) \rho-(n-1) \lambda)}{(n-1)|\nabla f|^{4}} \nabla \rho \cdot \nabla f-\frac{m^{2}+n-1}{(n-1)|\nabla f|^{2}} \rho^{2}+\frac{2 m}{|\nabla f|^{2}} \lambda \rho-\frac{n-1}{|\nabla f|^{2}} \lambda^{2} .
\end{aligned}
$$

Let $D_{i j k}=D\left(e_{i}, e_{j}, e_{k}\right)$. Then we have

$$
D_{i j k}=b_{1}\left(\nabla_{i} \rho \delta_{j k}-\nabla_{j} \rho \delta_{i k}\right)+b_{2}\left(\nabla_{i} f R_{j k}-\nabla_{j} f R_{i k}\right)+b_{3}\left(\nabla_{i} f \delta_{j k}-\nabla_{j} f \delta_{i k}\right) \text {, }
$$

where $\nabla_{i}=\nabla_{e_{i}}$ and

$$
b_{1}=-\frac{m}{2(n-1)(n-2)}, \quad b_{2}=\frac{1}{n-2}, \quad b_{3}=-\frac{(n-1) \lambda-m \rho}{(n-1)(n-2)} .
$$

So we have

$$
\begin{aligned}
|D|^{2}= & \sum_{i, j, k=1}^{n} D_{i j k}^{2} \\
= & b_{1}^{2}\left(2(n-1)|\nabla \rho|^{2}\right)+b_{2}^{2}\left(2|\nabla f|^{2}|\operatorname{Ric}|^{2}-2 \operatorname{Ric}^{2}(\nabla f, \nabla f)\right) \\
& +b_{3}^{2}\left(2(n-1)|\nabla f|^{2}\right)+2 b_{1} b_{2}(2 \operatorname{scal} \nabla \rho \cdot \nabla f-2 \operatorname{Ric}(\nabla f, \nabla \rho)) \\
& +2 b_{1} b_{3}(2(n-1) \nabla \rho \cdot \nabla f)+2 b_{2} b_{3}\left(2|\nabla f|^{2} \operatorname{scal}-2 \operatorname{Ric}(\nabla f, \nabla f)\right)
\end{aligned}
$$




$$
\begin{gathered}
=\frac{1}{2(n-1)(n-2)^{2}}\left(4(n-1)|\nabla f|^{2}|\mathrm{Ric}|^{2}-m^{2} n|\nabla \rho|^{2}\right) \\
+\frac{4 m((m+n-1) \rho-(n-1) \lambda)}{2(n-1)(n-2)^{2}}(\nabla \rho \cdot \nabla f) \\
-\frac{4\left(((n-1) \lambda-m \rho)^{2}+(n-1) \rho^{2}\right)}{2(n-1)(n-2)^{2}}|\nabla f|^{2} .
\end{gathered}
$$

A straightforward computation shows that

$$
|D|^{2}=\frac{2|\nabla f|^{4}}{(n-2)^{2}} \sum_{a, b=2}^{n}\left|h_{a b}-\sigma g_{a b}\right|^{2}+\frac{m^{2}}{2(n-1)(n-2)}\left|\nabla^{\Sigma} \rho\right|^{2} .
$$

Substituting the function $\rho$ by scal gives us the desired identity.

Similarly, the vanishing of the $D$-tensor implies many nice properties about the geometry of the warped product Einstein manifold $\left(M^{n}, g, f\right)$ and the level sets of $f$.

Proposition 3.3. Suppose $\left(M^{n}, g, f\right)(n \geq 3)$ is a $(\lambda, n+m)$-Einstein manifold with $m \neq 1$ and $D=0$. Let $c$ be a regular value of $f$ and $\Sigma=\{x \in M \mid f(x)=c\}$ be the level hypersurface of $f$. Then:

(1) Both the scalar curvature and $|\nabla f|^{2}$ are constant on $\Sigma$.

(2) On $\Sigma$, the Ricci tensor has either a unique eigenvalue or two distinct eigenvalues with multiplicity 1 and $n-1 ;$ moreover, the eigenvalue with multiplicity 1 is in the direction of $\nabla f$.

(3) The second fundamental form $h_{a b}$ of $\Sigma$ is of the form $h_{a b}=\frac{H}{n-1} g_{a b}$.

(4) The mean curvature $H$ is constant on $\Sigma$.

(5) $R(\nabla f, X, Y, Z)=0$ for any vectors $X, Y$, and $Z$ tangent to $\Sigma$.

Proof. It follows the argument in the proof of [Cao and Chen 2011, Proposition 3.1] by using Lemma 3.2.

Remark 3.4. If a $(\lambda, n+m)$-Einstein manifold with $m \neq 2-n$ has a harmonic Weyl tensor and $W(\nabla f, \cdot, \cdot, \cdot)$, then the $D$-tensor vanishes by Lemma 3.1. So Proposition 3.3 offers an alternative proof of [He et al. 2012, Theorem 7.9], which is the main step for the global classification in [ibid., Theorem 7.10].

\section{The proof of Theorems 1.1 and 1.2}

In this section, we first prove Theorem 1.2, which says that a compact Bach flat $(\lambda, n+m)$-Einstein manifold with $m \neq 0,1$, or $2-n$ has a harmonic Weyl tensor and $W(X, Y, Z, \nabla f)=0$ for any $X, Y, Z \in T M$. Then Theorem 1.1 follows by using [He et al. 2012, Theorem 7.9]. 
Proof of Theorem 1.2. We follow the argument in [Cao and Chen 2011]. Fix a point $p \in M$ and assume that $\left\{E_{i}\right\}_{i=1}^{n}$ is an orthonormal frame with $\nabla E_{i}(p)=0$. Using (2-3), the Bach tensor equation (2-4), and Lemma 3.1, a direct computation shows that for any $X, Y \in T M$, we have

$$
\begin{aligned}
(n-2) B(X, Y)= & \sum_{i}\left(\nabla_{E_{i}} C\right)\left(E_{i}, X, Y\right)+\sum_{i, j} \operatorname{Ric}\left(E_{i}, E_{j}\right) W\left(X, E_{i}, E_{j}, Y\right) \\
= & \left(\nabla_{E_{i}} W\right)\left(E_{i}, X, Y, \nabla f\right)+W\left(E_{i}, X, Y, \nabla_{E_{i}} \nabla f\right) \\
& \quad+\frac{m+n-2}{m}\left(\nabla_{E_{i}} D\right)\left(E_{i}, X, Y\right)+\operatorname{Ric}\left(E_{i}, E_{j}\right) W\left(X, E_{i}, E_{j}, Y\right) \\
= & (\operatorname{div} W)(\nabla f, Y, X)+\frac{m+n-2}{m}\left(\nabla_{E_{i}} D\right)\left(E_{i}, X, Y\right) \\
& \quad+W\left(X, E_{i}, E_{j}, Y\right)\left(\operatorname{Ric}\left(E_{i}, E_{j}\right)+\operatorname{Hess} f\left(E_{i}, E_{j}\right)\right) \\
= & \frac{n-3}{n-2} C(\nabla f, Y, X)+\frac{m+n-2}{m}\left(\nabla_{E_{i}} D\right)\left(E_{i}, X, Y\right) \\
& \quad+W\left(X, E_{i}, E_{j}, Y\right)\left(\frac{1}{m} g\left(\nabla f, E_{i}\right) g\left(\nabla f, E_{j}\right)+\lambda g\left(E_{i}, E_{j}\right)\right) \\
= & \frac{n-3}{n-2} C(\nabla f, Y, X)+\frac{m+n-2}{m}\left(\nabla_{E_{i}} D\right)\left(E_{i}, X, Y\right) \\
& \quad+\frac{1}{m} W(\nabla f, X, Y, \nabla f) .
\end{aligned}
$$

Letting $X=Y=\nabla f$ and integrating over $M$ yields

$$
\begin{aligned}
\frac{m(n-2)}{m+n-2} \int_{M} B(\nabla f, \nabla f) d \mathrm{vol} & =\int_{M} \sum_{i}\left(\nabla_{E_{i}} D\right)\left(E_{i}, \nabla f, \nabla f\right) d \mathrm{vol} \\
& =-\int_{M} \sum_{i} D\left(E_{i}, \nabla f, \nabla_{E_{i}} \nabla f\right) d \mathrm{vol} .
\end{aligned}
$$

For the integrand, using the fact that the $D$-tensor is trace-free for any two indices, we have

$$
\begin{aligned}
& -\sum_{i} D\left(E_{i}, \nabla f, \nabla_{E_{i}} \nabla f\right) \\
& \quad=\sum_{i, j} D\left(E_{i}, \nabla f, E_{j}\right)\left(\operatorname{Ric}\left(E_{i}, E_{j}\right)-\frac{1}{m} g\left(E_{i}, \nabla f\right) g\left(E_{j}, \nabla f\right)-\lambda g\left(E_{i}, E_{j}\right)\right) \\
& \quad=\sum_{i, j, k} D\left(E_{i}, E_{k}, E_{j}\right) \operatorname{Ric}\left(E_{i}, E_{j}\right) g\left(E_{k}, \nabla f\right) \\
& \quad=\frac{1}{2} \sum_{i, j, k} D\left(E_{i}, E_{k}, E_{j}\right)\left(\operatorname{Ric}\left(E_{i}, E_{j}\right) g\left(E_{k}, \nabla f\right)-\operatorname{Ric}\left(E_{k}, E_{j}\right) g\left(E_{i}, \nabla f\right)\right) \\
& =-\frac{1}{2} \sum_{i, j, k}\left|D\left(E_{i}, E_{j}, E_{k}\right)\right|^{2} .
\end{aligned}
$$


It follows that

$$
\frac{m(n-2)}{m+n-2} \int_{M} B(\nabla f, \nabla f) d \mathrm{vol}=-\frac{1}{2} \int_{M}|D|^{2} d \mathrm{vol} .
$$

So a vanishing Bach tensor implies that the $D$-tensor vanishes on $M$.

From (3-1), we have $C(X, Y, Z)=W(X, Y, Z, \nabla f)$. We show that both are zero on the regular points of $f$, and thus on $M$, since $f$ is an analytic function (see [He et al. 2012, Proposition 2.8]). At a regular point of $f$, we choose $E_{1}=\nabla f /|\nabla f|$ and let $C_{i j k}=C\left(E_{i}, E_{j}, E_{k}\right)$. By the symmetry of the Weyl tensor, we have $C_{i j 1}=0$. Let $a, b, c \geq 2$ be integers. From Proposition 3.3, we have $\operatorname{Ric}\left(E_{1}, E_{a}\right)=0$ and $R\left(E_{1}, E_{a}, E_{b}, E_{c}\right)=0$, and thus $W\left(E_{a}, E_{b}, E_{c}, E_{1}\right)=R\left(E_{a}, E_{b}, E_{c}, E_{1}\right)=0$. So we have $C_{a b c}=W\left(E_{a}, E_{b}, E_{c}, \nabla f\right)=0$. It remains to show that $C_{1 i j}=0$ for any $i, j=1, \ldots, n$. Since $D=0$, Bach flatness implies that

$$
\begin{aligned}
0=(n-2) B\left(E_{i}, E_{j}\right) & =\frac{n-3}{n-2} C_{1 i j}|\nabla f|+\frac{1}{m} W\left(E_{1}, E_{i}, E_{j}, \nabla f\right)|\nabla f| \\
& =\frac{n-3}{n-2} C_{1 i j}|\nabla f|+\frac{1}{m} C_{1 i j}|\nabla f| .
\end{aligned}
$$

It follows that $C_{1 i j}=0$ if $m \neq-(n-2) /(n-3)$. We have $-(n-2) /(n-3)=-2$ when $n=4$, which is excluded in the theorem. When $n \geq 5$, an extension of [Cao and Chen 2011, Proposition 5.1] shows that $C_{1 i j}=0$ for all $m \neq 0,1$, or $2-n$.

Proof of Theorem 1.1. From Theorem 1.2, we know that $\left(M^{4}, g, f\right)$ has a harmonic Weyl tensor and $W(\nabla f, X, Y, Z)=0$ for any $X, Y, Z \in T M$. We assume that $M$ is not Einstein. At a regular point $p$ of $f$, we assume that the Ricci tensor has distinct eigenvalues. The complement of such points can not contain an open set, as $g$ and $f$ are analytic in the harmonic coordinate (see [He et al. 2012, Proposition 2.8]). So it is enough to show that the metric $g$ is locally conformally flat around $p$. [He et al. 2012, Theorem 7.9] says that the metric is locally a warped product over an interval, meaning that $g=d t^{2}+\psi(t)^{2} g_{L}$, where $\left(L^{3}, g_{L}\right)$ is an Einstein metric and thus has constant curvature. A computation shows that such a metric has vanishing Weyl tensors, i.e., that it is locally conformally flat.

An alternative approach is to use the symmetries of Weyl tensors to show that they are zeros, as in the proof of [Cao and Chen 2011, Theorem 1.1].

Remark 4.1. In [He et al. 2012], the authors considered a warped product Einstein manifold with a nonempty boundary. Let $w=\exp (-f / m)$ in the interior of $M$ and $w=0$ on the boundary $\partial M$. Both Theorem 1.1 and Theorem 1.2 can also be extended to the case when $M$ has a nonempty boundary. For any small $\epsilon>0$, we define $M_{\epsilon}=\{x \in M: w(x) \geq \epsilon\}$, and we only have to show that $D=0$ on $M_{\epsilon}$. Then taking the limit $\epsilon \rightarrow 0$ implies that $D=0$ on $M$. In fact, the boundary term 
of the integral (4-1) vanishes:

$$
\int_{\partial M_{\epsilon}} D(v, \nabla f, \nabla f) d \mathrm{vol}=0,
$$

since the unit normal vector $v$ of $\partial M_{\epsilon}$ is parallel to $\nabla f$. So the integral equation (4-2) holds on $M_{\epsilon}$, and then $D=0$ on $M_{\epsilon}$.

\section{Acknowledgements}

The authors would like to thank Huai-Dong Cao for enlightening conversations and helpful suggestions. We also thank the anonymous referees for their valuable comments on our previous version.

\section{References}

[Bach 1921] R. Bach, "Zur Weylschen Relativitätstheorie und der Weylschen Erweiterung des Krümmungstensorbegriffs", Math. Z. 9:1-2 (1921), 110-135. MR 1544454 JFM 48.1035.01

[Besse 1987] A. L. Besse, Einstein manifolds, Ergebnisse der Mathematik und ihrer Grenzgebiete (3) 10, Springer, Berlin, 1987. MR 88f:53087 Zbl 0613.53001

[Böhm 1998] C. Böhm, "Inhomogeneous Einstein metrics on low-dimensional spheres and other low-dimensional spaces", Invent. Math. 134:1 (1998), 145-176. MR 99i:53046 Zbl 0965.53033

[Cao 2010] H.-D. Cao, "Recent progress on Ricci solitons", pp. 1-38 in Recent advances in geometric analysis, edited by Y.-I. Lee et al., Adv. Lect. Math. (ALM) 11, International Press, Somerville, MA, 2010. MR 2011d:53061 Zbl 1201.53046

[Cao and Chen 2011] H.-D. Cao and Q. Chen, "On Bach-flat gradient shrinking Ricci solitons", preprint, 2011. arXiv 1105.3163v3

[Cao and Chen 2012] H.-D. Cao and Q. Chen, "On locally conformally flat gradient steady Ricci solitons”, Trans. Amer. Math. Soc. 364:5 (2012), 2377-2391. MR 2888210 Zbl 1245.53038

[Case et al. 2011] J. Case, Y.-J. Shu, and G. Wei, "Rigidity of quasi-Einstein metrics", Differential Geom. Appl. 29:1 (2011), 93-100. MR 2012d:53124 Zbl 1215.53033

[Catino 2012] G. Catino, "A note on four-dimensional (anti-)self-dual quasi-Einstein manifolds", Differential Geom. Appl. 30:6 (2012), 660-664. MR 2996860 Zbl 06126062

[Catino et al. 2011] G. Catino, C. Mantegazza, L. Mazzieri, and M. Rimoldi, "Locally conformally flat quasi-Einstein manifolds", preprint, 2011. arXiv 1010.1418v3

[He et al. 2010] C. He, P. Petersen, and W. Wylie, "Warped product Einstein metrics over spaces with constant scalar curvature", preprint, 2010. arXiv 1012.3446v1

[He et al. 2011] C. He, P. Petersen, and W. Wylie, "Uniqueness of warped product Einstein metrics and applications", preprint, 2011. arXiv 1110.2456

[He et al. 2012] C. He, P. Petersen, and W. Wylie, "On the classification of warped product Einstein metrics”, Comm. Anal. Geom. 20:2 (2012), 271-311. MR 2928714

[Kim and Kim 2003] D.-S. Kim and Y. H. Kim, "Compact Einstein warped product spaces with nonpositive scalar curvature”, Proc. Amer. Math. Soc. 131:8 (2003), 2573-2576. MR 2004b:53063 Zbl 1029.53027

[Qian 1997] Z. Qian, "Estimates for weighted volumes and applications", Quart. J. Math. Oxford Ser. (2) 48:190 (1997), 235-242. MR 98e:53058 Zbl 0902.53032 
[Villani 2009] C. Villani, Optimal transport: Old and new, Grundlehren der Mathematischen Wissenschaften 338, Springer, Berlin, 2009. MR 2010f:49001 Zbl 1156.53003

[Wei and Wylie 2009] G. Wei and W. Wylie, "Comparison geometry for the Bakry-Emery Ricci tensor”, J. Differential Geom. 83:2 (2009), 377-405. MR 2011a:53064 Zbl 1189.53036

Received July 5, 2012. Revised December 3, 2012.

\section{QIANG CHEN}

DEPARTMENT OF MATHEMATICS

LEHIGH UNIVERSITY

BETHLEHEM, PA 18015

UNITED STATES

qic208@lehigh.edu

ChenXu He

DEPARTMENT OF MATHEMATICS

LEHIGH UNIVERSITY

BETHLEHEM, PA 18015

United STATES

Current address:

DEPARTMENT OF MATHEMATICS

UNIVERSITY OF OKLAHOMA

NORMAN, OK 73019

UNITED STATES

he.chenxu@ou.edu 


\title{
PACIFIC JOURNAL OF MATHEMATICS
}

\author{
msp.org/pjm
}

Founded in 1951 by E. F. Beckenbach (1906-1982) and F. Wolf (1904-1989)

\section{EDITORS}

V. S. Varadarajan (Managing Editor)

Department of Mathematics

University of California

Los Angeles, CA 90095-1555

pacific@math.ucla.edu

Paul Balmer

Department of Mathematics

University of California

Los Angeles, CA 90095-1555

balmer@math.ucla.edu

Daryl Cooper

Department of Mathematics

University of California

Santa Barbara, CA 93106-3080 cooper@math.ucsb.edu

Jiang-Hua $\mathrm{Lu}$

Department of Mathematics

Pokfulam Rd., Hong Kong jhlu@maths.hku.hk
The University of Hong Kong

Don Blasius

Department of Mathematics University of California

Los Angeles, CA 90095-1555

blasius@math.ucla.edu

Robert Finn

Department of Mathematics Stanford University

Stanford, CA 94305-2125

finn@math.stanford.edu

Sorin Popa

Department of Mathematics

University of California

Los Angeles, CA 90095-1555 popa@math.ucla.edu

Paul Yang

Department of Mathematics Princeton University

Princeton NJ 08544-1000

yang@math.princeton.edu

\section{PRODUCTION}

Silvio Levy, Scientific Editor, production@msp.org

\section{SUPPORTING INSTITUTIONS}

ACADEMIA SINICA, TAIPEI

CALIFORNIA INST. OF TECHNOLOGY

INST. DE MATEMÁTICA PURA E APLICADA

KEIO UNIVERSITY

MATH. SCIENCES RESEARCH INSTITUTE

NEW MEXICO STATE UNIV.

OREGON STATE UNIV.

\author{
STANFORD UNIVERSITY \\ UNIV. OF BRITISH COLUMBIA \\ UNIV. OF CALIFORNIA, BERKELEY \\ UNIV. OF CALIFORNIA, DAVIS \\ UNIV. OF CALIFORNIA, LOS ANGELES \\ UNIV. OF CALIFORNIA, RIVERSIDE \\ UNIV. OF CALIFORNIA, SAN DIEGO \\ UNIV. OF CALIF., SANTA BARBARA
}

\author{
Vyjayanthi Chari \\ Department of Mathematics \\ University of California \\ Riverside, CA 92521-0135 \\ chari@math.ucr.edu \\ Kefeng Liu \\ Department of Mathematics \\ University of California \\ Los Angeles, CA 90095-1555 \\ liu@math.ucla.edu \\ Jie Qing \\ Department of Mathematics \\ University of California \\ Santa Cruz, CA 95064 \\ qing@cats.ucsc.edu
}

These supporting institutions contribute to the cost of publication of this Journal, but they are not owners or publishers and have no responsibility for its contents or policies.

See inside back cover or msp.org/pjm for submission instructions.

The subscription price for 2013 is US \$400/year for the electronic version, and \$485/year for print and electronic.

Subscriptions, requests for back issues and changes of subscribers address should be sent to Pacific Journal of Mathematics, P.O. Box 4163, Berkeley, CA 94704-0163, U.S.A. The Pacific Journal of Mathematics is indexed by Mathematical Reviews, Zentralblatt MATH, PASCAL CNRS Index, Referativnyi Zhurnal, Current Mathematical Publications and the Science Citation Index.

The Pacific Journal of Mathematics (ISSN 0030-8730) at the University of California, c/o Department of Mathematics, 798 Evans Hall \#3840, Berkeley, CA 94720-3840, is published twelve times a year. Periodical rate postage paid at Berkeley, CA 94704, and additional mailing offices. POSTMASTER: send address changes to Pacific Journal of Mathematics, P.O. Box 4163, Berkeley, CA 94704-0163.

PJM peer review and production are managed by EditFLOW ${ }^{\circledR}$ from Mathematical Sciences Publishers.

\section{PUBLISHED BY}

mathematical sciences publishers

nonprofit scientific publishing

http://msp.org/

(C) 2013 Mathematical Sciences Publishers 


\section{PACIFIC JOURNAL OF MATHEMATICS}

Volume $265 \quad$ No. $2 \quad$ October 2013

Singularity removability at branch points for Willmore surfaces

YANN BERNARD and TRISTAN RIVIÈRE

On Bach flat warped product Einstein manifolds

QIANG CHEN and CHENXU HE

On plane sextics with double singular points

Alex DeGTyareV

A computational approach to the Kostant-Sekiguchi correspondence

HEIKo DiETRICH and WILLEM A. DE GRAAF

Landau-Toeplitz theorems for slice regular functions over quaternions

GRAZIANO GENTILI and GiUlia SARFATTI

On surgery curves for genus-one slice knots

PATRICK M. GILMER and CHARLES LIVINGSTON

Characterizing abelian varieties by the reduction of the Mordell-Weil group

Chris Hall and Antonella Perucca

Variation of complex structures and the stability of Kähler-Ricci

Solitons

StUART J. HALl and ThOMAS MURPHY

On crossed homomorphisms of the volume preserving diffeomorphism groups

RYOJI KASAGAWA

Regularity at the boundary and tangential regularity of solutions of the Cauchy-Riemann system

TRAN VU KHANH and GIUSEPPE ZAMPIERI

On the Steinberg character of a semisimple $p$-adic group 